\title{
Method of autofluorescence diagnostics of skin neoplasms in the near infrared region
}

\author{
Yulia A. Khristoforova ${ }^{1 *}$, Ivan A. Bratchenko ${ }^{1}$, Dmitry N. Artemyev ${ }^{1}$, Oleg O. Myakinin ${ }^{1}$, \\ Alexander A. Moryatov ${ }^{2}$, Sergey V. Kozlov ${ }^{2}$, Valery P. Zakharov ${ }^{1}$ \\ ${ }^{1}$ Laser and biotechnical systems department, Samara State Aerospace University, \\ 34 Moskovskoe Shosse, Samara 443086, Russia \\ 2 Oncology Department, Samara State Medical University, 89 Chapaevskaya str., Samara 443099, Russia \\ *e-mail: khristoforovajulia@gmail.com
}

\begin{abstract}
We propose a method for the diagnostics of skin neoplasms, based on the analysis of the changes in the autofluorescence spectra in the near infrared range. The autofluorescence was excited by means of the laser radiation with the wavelength 785 $\mathrm{nm}$ for ex vivo and in vivo studies with subsequent exponential approximation of its spectrum. The quantitative and qualitative criteria for the neoplasm type differentiation by the change of the curvature and the rate of decrease of the approximating curve are found. It is shown that the proposed approach allows the diagnostics of malignant melanomas with the accuracy of $88.4 \%$ for ex vivo studies, and $86.2 \%$ for in vivo ones. (C) 2015 Samara State Aerospace University (SSAU).
\end{abstract}

Keywords: autofluorescence, near IR range, dermatology, melanoma, exponential approximation, sensitivity, specificity.

Paper \#2887 received 2015.12.02; accepted for publication 2015.12.20; published online 2015.12.29.

\section{References}

1. P. Bourne, C. Rosendabl, J. Keir, and A. Cameron, "A diagnostic algorithm for skin cancer diagnosis combining clinical features with dermatoscopy findings," Research Dermatol. Pract. Concept. 2(2), 55-61 (2012).

2. M. Mogensen, L. Thrane, and T. M. Jorgensen, "OCT imaging of skin cancer and other dermatological diseases," J. Biophotonics 2(6-7), 442-451 (2009).

3. M. Mogensen, B. M. Nürnberg, J. L. Forman, J. B. Thomsen, L. Thrane, and G. B. E. Jemec, "In vivo thickness measurement of basal cell carcinoma and actinic keratosis with optical coherence tomography and 20MHz ultrasound," Brit. J. Dermatol. 160(5), 1026-1033 (2009).

4. N. Aspres, I. B. Egerton, and A. C. Lim, "Imaging the skin,” Austral. J. Dermatol. 44(1), 19-27 (2003).

5. H. Lui, J. Zhao, D. McLean, and H. Zeng, "Real-time Raman spectroscopy for in vivo skin cancer diagnosis," Cancer Res. 72(10), 2491-2500 (2012).

6. V. P. Zakharov, I. A. Bratchenko, D. N. Artemyev, O. O. Myakinin, D. V. Kornilin, S. V. Kozlov, and A. A. Moryatov , "Comparative analysis of combined spectral and optical tomography methods for detection of skin and lung cancers,” J. Biomed. Opt. 20(2), 025003 (2015).

7. J. Zhao, H. Lui, D. I. McLean, and H. Zeng, "Automated autofluorescence background subtraction algorithm for biomedical Raman spectroscopy,” Applied Spectroscopy 61(11), 1225-1232 (2007).

8. E. G. Borisova, L. P. Angelova, and E. P. Pavlova, "Endogenous and Exogenous Fluorescence Skin Cancer Diagnostics for Clinical Applications," IEEE J of Selected Topics in Quantum Electronics 20(2), 7100412 (2014).

9. N. Baletic, H. Malicevic, Z. Petrovic, J. Marinkovic-Eric, and A. Peric, "Advantages and limitations of the autofluorescent diagnostics of the laryngeal cancer and precancerosis," Eur. Arch. Otorhinolaryngol. 267(6), 925-931 (2010).

10. V. P. Zakharov, I. A. Bratchenko, D. N. Artemyev, O. O. Myakinin, D. V. Kornilin, S. V. Kozlov, and A. A. Moryatov, "Lung neoplasm diagnostics using Raman spectroscopy and autofluorescence analysis," J. Biomed. Photonics and Eng. 1(1), 70-6 (2015). 
11. W. Lo, Y. L. Chang, Y. Sun, S. J. Lin, and S. H. Jee, "In-vitro visualization of corneal wound healing in an organ culture model using multiphoton autofluorescence and second harmonic generation microscopy," Proc. of SPIE 6426, 642617 (2007).

12. L. Rovati, and F. Docchio, “Autofluorescence methods in ophthalmology,” J. Biomed. Opt. 9(1), 9-21 (2004).

13. E. G. Borisova, E. Nikolova, P. P. Troyanova, and L. A. Avramov, "Autofluorescence and diffuse reflectance spectroscopy of pigment disorders in human skin," Journal of Optoelectronics and Advanced Materials 10(3), 717-722 (2008).

14. Z. Huang, H. Zeng, I. Hamzavi, D. McLean, and H. Lui, "Rapid near-infrared Raman spectroscopy system for real-time in vivo skin measurements," Opt. Lett. 26(22), 1782-1784 (2001).

15. J. A. Nelder, and R. Mead, "A simple method for function minimization," Computer Journal 7, 308-313 (1965).

16. G. M. Fikhtengol'ts, The fundamentals of mathematical analysis: International series in pure and applied mathematics, 1st ed., Pergamon (1965). ISBN: 978-0080134734

17. D. Y. Churmakov, I. V. Meglinski, S. A. Piletsky, and D. A. Greenhalgh, "Analysis of skin tissues spatial fluorescence distribution by the Monte Carlo simulation," Journal of Physics D: Applied Physics 36(14), 17221728 (2003).

18. S. K Majumder., M. D. Keller, F. I. Boulos, M. C. Kelley, and A. Mahadevan-Jansen, "Comparison of autofluorescence, diffuse reflectance, and Raman spectroscopy for breast tissue discrimination," J. Biomed. Opt. 13(5), 054009 (2008).

19. V. V. Tuchin, Tissue optics: Light Scattering Methods and Instruments for Medical Diagnosis, 3rd ed., SPIE Press, Washington Bellingham, (2015). ISBN: 9781628415162.

20. S. Wang, J. Zhao, H. Lui, Q. He, and H. Zeng, "In vivo near infrared autofluorescence imaging of pigmented skin lesions: methods, technical improvements and preliminary clinical results," Skin Research and Technology 19(1), 20-26 (2013).

21. A. B. Pravdin, S. R. Utz, A. A. Al'khov, and A. N. Bashkatov, "Upper epidermis autofluorescence dynamics under laser UV irradiation," Proc. SPIE 2100, 233-236 (1994).

22. L. Lim, B. Nichols, M. Midgen, N. Rajaram, J. Reichenberg, M. Markey, M. Ross, and J. Tunnell, "Clinical study of noninvasive in vivo melanoma and nonmelanoma skin cancers using multimodal spectral diagnosis," J. Biomed. Opt.19(11), 117003 (2014).

23. E. Borisova, D. Dogandjiiska, I. Bliznakovaa, L. Avramova, E. Pavlova, and P. Troyanovad, "Multispectral autofluorescence diagnosis of non-melanoma cutaneous tumors," Proc. of SPIE-OSA 7368, 736823 (2009).

24. E. Borisova, P. Troyanova, P. Pavlova, L. and Avramov, "Diagnostic pigment skin neoplasms by laserinduced autofluorescence and diffusely reflectance spectroscopy," Quantum Electronics 38(6), 597-605 (2008).

\section{Introduction}

The skin cancer is one of the most serious problems of the humanity, which is characterised by the high mortality index and treatment complexity. This disease can occur in any humans independent of sex, age and other specific features. Although the skin neoplasms can be revealed even by a visual examination, their diagnostics is very complex, and only $40 \%$ of the disease cases can be diagnosed during the first visit to an experienced physician [1]. The "golden standard" of unambiguous identification of the neoplasm type in the oncology is the histologic examination that requires the sample biopsy. This procedure can lead to such complications as bleeding, infection, persisting wounds, etc. If the skin melanoma is suspected, the preoperational biopsy of the neoplasm is contra-indicated, since it can cause dramatic growth of the melanoma and active metastasis. In this regard, an urgent necessity exists in the development of instrumental methods of noninvasive diagnostics of skin neoplasms.

To increase the diagnostics efficiency and the quality of tumour treatment it is reasonable to use the optical noninvasive methods of the tissue diagnostics. The modern method of confocal laser scanning microscopy is still not available in clinics because of high cost of the instrumentation [2]. The method of optical coherence tomography (OCT) offers the possibility to visualise the internal structure of the skin and provides high accuracy of diagnosing the basal cell carcinoma due to visualising the so called "nests" in the tumour structure, but it does not allow the diagnostics of melanoma with high accuracy [3]. Various imaging methods allow only the analysis of the spatial localisation of the neoplasm [4]. The Raman scattering is a high-sensitive method for identifying the melanoma [5]. However, since the Raman scattering probability is small, the signal acquisition takes much time and the diagnostics accuracy decreases in the mass screening. It is possible to increase the accuracy of the diagnostics using complex approaches combining a few optical diagnostic methods [6].

It is interesting to note that during the Raman scattering registration, the fluorescence component of the scattered radiation is usually eliminated as low-informative [7]. However, the fluorescence response of a tissue to the laser irradiation can be also used to identify the 
neoplasm type [8], since the autofluorescence (AF) in the near infrared range carries information about the quantitative and qualitative changes of the composition of natural fluorophores in the area of neoplasms in comparison with the surrounding normal skin. This approach is free of the restrictions related to the time of the signal acquisition, inherent in the Raman scattering method, and admits the real-time operation without damaging the studied tissue, which allows for fast and economical solution of medical practice problems. The application of AF method was demonstrated in a number of papers for the cancer diagnostics in larynx [9] and lung [10]. The AF spectroscopy was also applied in wound healing monitoring [11] and eye tissue studies [12].

The concentration of natural fluorophores in the skin is very personal and can vary within a wide interval of values, which leads to an essential dispersion of the values of the AF intensity and the time of its decay from patient to patient. This does not allow the use of threshold methods of neoplasm diagnostics, which are characterised by low efficiency [13]. In the present paper, it is proposed to use the features of the AF spectra deformations in neoplasms as compared to the AF spectra of the surrounding normal skin as the informative criteria. The assessment of the AF curve behaviour was implemented using the exponential approximation of the studied curve.

\section{Materials and methods}

\subsection{Experimental setup}

The experimental studies were performed using the setup schematically presented in Fig. 1. The setup incorporates the thermally stabilised semiconductor laser unit LML-785.0RB-04 with the radiation wavelength $785 \mathrm{~nm}$, the spectrograph Shamrock SR$303 \mathrm{i}$ with the integrated digital camera ANDOR DV420A-OE, the optical unit, and the PC.

The probing laser radiation is passed through the bandpass filter BPF, directed by the mirror $\mathrm{M}$ and the dichroic reflector DM and focused by the lens $\mathrm{L}_{2}$ onto the studied sample. The filter BPF served to protect the studied sample from the radiation of Raman scattering that occurs in the entrance optical fibre. The scattered radiation including the Rayleigh scattering, and the fluorescence signal was collected by the lens $\mathrm{L}_{2}$ and sent to the spectrometer through a broadband filter LPF, aimed at filtering the probing laser radiation. The lenses $\mathrm{L}_{1}$ and $\mathrm{L}_{2}$ were used to match the optical unit with the entrance and collection optical fibre.

The signal was recorded within the spectral range 775$925 \mathrm{~nm}$ with the resolution $0.05 \mathrm{~nm}$ and low level of intrinsic noise. The radiation power did not exceed 200 $\mathrm{mW}$. The used laser radiation of the near infrared region with the wavelength $785 \mathrm{~nm}$ provides deep penetration into the tissue by $\sim 4 \mathrm{~mm}$ without damaging the integrity of the skin [14].

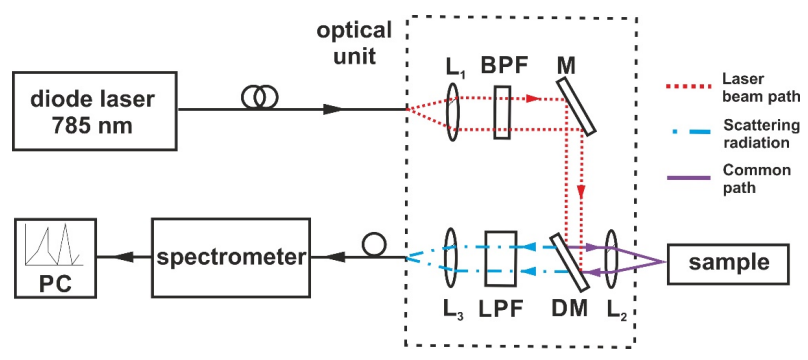

Fig. 1 Schematic diagram of the experimental setup.

\subsection{Tissue samples}

The in vivo studies of 28 human skin areas were carried out, including the AF spectra recording of tumours and surrounding healthy skin (14 melanoma areas, 9 areas of basal cell carcinoma, 1 area of squamous cess carcinoma, 3 areas of nevus, and 1 area of benign tumour). The ex vivo studies were carried out for 88 human skin samples with the registration of the tumour and surrounding healthy skin (34 samples of melanoma, 35 samples of basal cell carcinoma, 9 samples of benign tumours, 4 samples of squamous cell carcinoma, 3 samples of nevus, and 3 samples of skin cancer).

All tissue samples for the ex vivo studies were obtained as a result of surgical extraction from the patients of the Samara Regional Clinical Oncologic Dispensary and studied not later than in 4 hours after the resection. Each sample was a tumour surrounded by a healthy tissue. The size of the studied neoplasms varied in a wide interval from $0.5 \mathrm{~cm}$ to $3.5 \mathrm{~cm}$. The diagnosis for each neoplasm was confirmed by the result of histologic examination. All in vivo and ex vivo studies were performed after the patients agreement. The ethic committee of Samara State Medical University approved the investigations.

\subsection{Method of exponential approximation}

The AF spectra of each sample were recorded in the neoplasm area and in the healthy skin area adjacent of pathology region. Within the spectral range $870-920$ $\mathrm{nm}$ the intensity of AF decreases with the growth of the wavelength, and the most significant qualitative changes in the AF spectrum were observed within the range 810 - $870 \mathrm{~nm}$. For the comparative analysis of the experimental data in the above range we approximate the AF spectrum with an exponential function:

$$
I_{a p}(\lambda)=a e^{b\left(\frac{\lambda}{\lambda_{\max }}\right)}+c
$$

where the sign of the coefficient $a$ is responsible for the shape (convex/concave) of the approximating curve, the coefficient $b$ characterises the curvature, and $\lambda_{\text {max }}=$ 870 is the right boundary of the approximation interval. The approximation was implemented using the criterion of minimising the root-mean-square error. For the minimisation algorithm (the search for the global minimum of the functional) we used the Nelder-Mead simplex-method and the stochastic method of 
differential evolution [15]. The limitation of 1000 iterations was imposed on each method. For the final result we took the values providing the minimal rootmean-square error of approximation. The mean relative error of approximation (with respect to the maximum of the spectrum in the approximated region) amounted to $2.3 \%$ for the melanoma spectra, $1.2 \%$ for the spectra of healthy skin, $1.9 \%$ for the spectra of basal cell carcinoma.

Generally, the curvature $k(\lambda)$ of the approximating curve of the spectral intensity (1) in the interval $\lambda \in[810 ; 870]$ is determined as [15]

$$
k(\lambda)=\frac{I_{a p}^{\prime \prime}(\lambda)}{\left(1+\left[I_{a p}^{\prime}(\lambda)\right]^{2}\right)^{3 / 2}}
$$

For convenience let us redefine the variable $\lambda$ as $\lambda \stackrel{\text { def }}{=} 870 \lambda, \lambda \in[810 / 870 ; 1]$. Then

$$
k(\lambda)=\frac{a b^{2} \exp (b \lambda)}{\left(1+a^{2} b^{2} \exp (2 b \lambda)\right)^{3 / 2}}
$$

The function $a^{2} b^{2} \exp (2 b \lambda) \geq 0$ for any $a, b, \lambda$ and is monotonic. Therefore,

$$
\begin{gathered}
|k(\lambda)|<|\hat{k}(\lambda)|, \\
\hat{k}(\lambda)=\frac{a b^{2} \exp (b \lambda)}{\left(a^{2} b^{2} \exp (2 b \lambda)\right)^{\frac{3}{2}}}= \\
=\frac{\operatorname{sign}(a)}{a^{2}|b|} \exp (-2 b \lambda) .
\end{gathered}
$$

The function $\hat{k}(\lambda)$ corresponds to the more stringent condition $a^{2} b^{2} \exp (2 b \lambda) \geq 1$, which was fulfilled for nearly all the neoplasms studied in this research. In this case, one can neglect the contribution of 1 in the denominator of Eq. (3), after which the function $k(\lambda)$ is reduced to $\hat{k}(\lambda)$ with similar properties.

\section{Results and discussion}

Due to the multilayer and multicomponent structure of the skin, its interaction with light is a complex process that includes the multiple scattering and absorption of photons. The quantum yield of the fluorescence of skin natural fluorophores varies from thousandth to hundredth parts of the absorbed radiation energy [17]. In healthy skin tissues, the main substances that actively absorb and scatter light are haemoglobin, water, lipids, and melanin [18]. The cell membranes, nuclei, organelles, melanin granules in the cells are the main scattering objects in a tissue. Depending on the malignant transformation of the tissue neoplasm, the randomness of the cell structure growth increases and the size of the cell nuclei grows. Thus, the mean value of the normal cell nucleus is $10-12 \mu \mathrm{m}$, for pathologic tissues it increases to $20-50 \mu \mathrm{m}$. The refractive index at the nucleus-cytoplasm boundary also increases [19].
All these features of the pathology development lead to the change of scattering and fluorescence characters in neoplasm tissues.

Beyond $800 \mathrm{~nm}$, the $\mathrm{AF}$ spectrum is a nonlinear decreasing function. The characteristic AF spectra of different skin tissues are shown in Fig. 2. It is seen that the autofluorescence intensity is essentially different for different types of pathology and the healthy skin.

Since the AF intensity is determined by the skin phenotype of a particular patient, to get the unified results that could be used in practice we analysed the quantities, normalised to the maximal value of the intensity of spectrum of the surrounding healthy skin for each sample, rather than the absolute AF intensities.

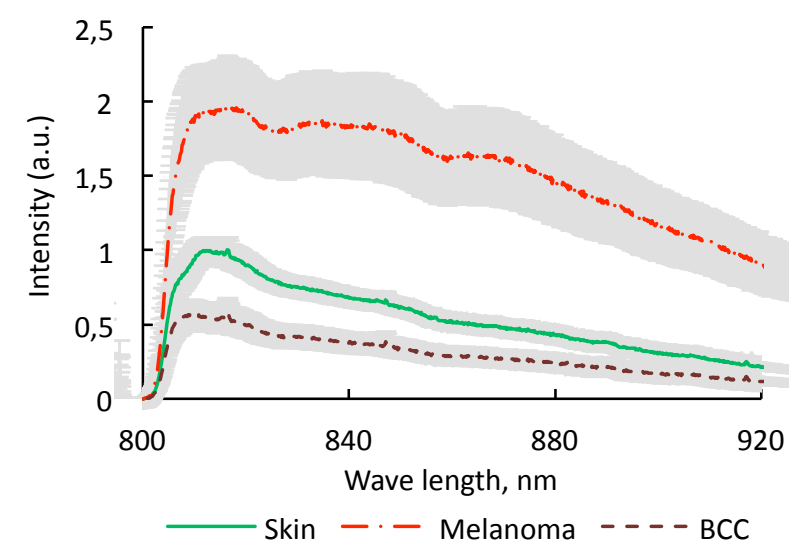

Fig. 2 AF spectra of healthy human skin and neoplasms.

In the course of the studies, it was found that the intensity of the AF spectrum in the area of basal cell carcinoma is reduced by $30-60 \%$ as compared to the intensity from the surrounding healthy skin area, and in the melanoma region, on the contrary, the AF intensity increases to $170-250 \%$. The AF spectra of the studied tissues are characterised by the presence of expressed local maxima in the region $800-860 \mathrm{~nm}$. These features of the AF spectra are related to the higher concentration of melanocytes and keratinocytes (containing melanin) in the tissues of melanomas and nevi as compared to the normal skin and basal cell carcinoma. The melanin intensely fluoresces in the near infrared region [20], which explains the high intensity of AF from pigmented formations.

In Fig 2 the plots of AF spectra are presented together with the confidence intervals of their measurement. One can see that the maximal spread of values of the AF spectrum intensity does not exceed 7\% for healthy skin, $12 \%$ for basal cell carcinoma, whereas for the melanoma it is significantly higher and amounts to $30-40 \%$. The decrease of the AF intensity is related to the continuous irradiation of the sample in the course of recording the AF spectrum, due to which the effect of the skin photobleaching occurs [21]. The photobleaching phenomenon arises due to the 
degradation of fluorophores because of photochemical damage and modification of molecular bonds. As seen from Fig. 1, the areas of possible AF responses from the healthy skin, melanoma and basal cell carcinoma do not intersect, which allows their differentiation by the level of intensity. However the situation is opposite in the case of melanocyte neoplasms (melanoma, nevus, moles) for which a high level of AF is typical (Fig. 3). In this case, the accuracy of melanocyte neoplasms differentiation is essentially reduced, if the intensity values are used as a threshold diagnostic criterion. Thus in our studies the accuracy of differentiating melanomas and nevi by the intensity did not exceed 52\%. In Ref. [13] the higher accuracy of differentiation of melanomas and nevi (about $80 \%$ ) by the AF intensity level in the visible region of the spectrum was achieved due to using the high-intensity UV sources of excitation and careful positioning of the optical sensor to minimise the error of detecting the AF signals. Guaranteeing the unchanged geometry of the AF intensity measurement in the neoplasms from different part of body having different shape and size requires additional effort and is difficult for clinical applications, particularly in screening and prophylactic examinations. To overcome these difficulties we propose a method based on the skin neoplasm differentiation only on the shape of the AF spectrum, giving up the threshold criteria of $\mathrm{AF}$ radiation intensity. This allows essentially faster measurement, which is of particular importance for in vivo applications.

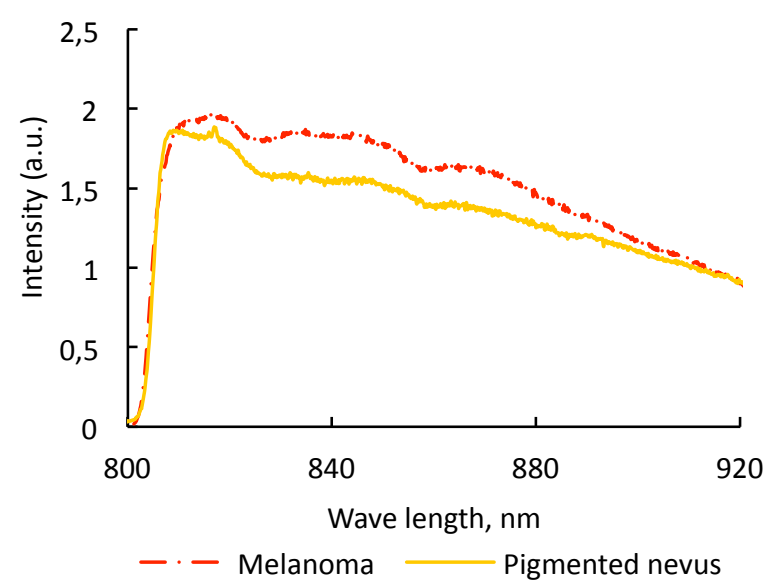

Fig. 3 AF spectra of malignant melanoma and pigmented nevus.

For the comparative analysis of the experimental data in the range $810-870 \mathrm{~nm}$ we approximated the AF spectral intensity by the exponential function (1): $I_{a p}(\lambda)=a e^{b\left(\frac{\lambda}{\lambda_{\max }}\right)}+c$.

As shown above, the sign (concave/convex) of the approximating function is completely determined by the sign of the parameter $a$ from Eq. (1). The parameter $c$ does not change the shape of the curve and, therefore, obviously is not involved in the diagnostic algorithm.
As follow from Eq. (1), the parameter $b$ is responsible for the "degree of nonlinearity". It should be noted that for the considered wavelength range in the case of exciting the skin fluorescence with the laser wavelength $785 \mathrm{~nm}$ only three cases are physically implementable (except all parameters are zero):

1. $a>0, b<0 \quad-$ concave monotonically decreasing function (1);

2. $a<0, b<0 \quad-\quad$ convex monotonically increasing function (1);

3. $a<0, b>0-$ convex monotonically decreasing function (1).

The first case describes concave rapidly decreasing functions, typical for the AF spectra of nonmelanocytic neoplasms (benign tumours, squamous cell cancer, and basal cell carcinoma). The case 3 $(a<0, b>0)$ characterises the AF spectra of melanoma and nevus. At the expense of the increased melanin concentration the melanocytic neoplasms more intensely emit the radiation in the considered range, therefore, the AF spectra of the melanocytic neoplasms are characterised by the convex shape and slow decrease. For example, the results of approximating the AF spectra are presented for the basal cell carcinoma and the melanoma in Fig. 4.

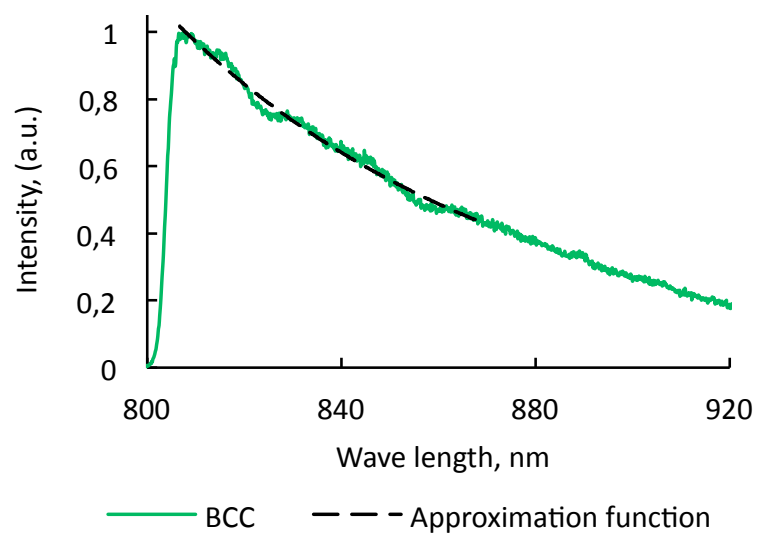

a) $a=6053.6 ; b=-0.64$

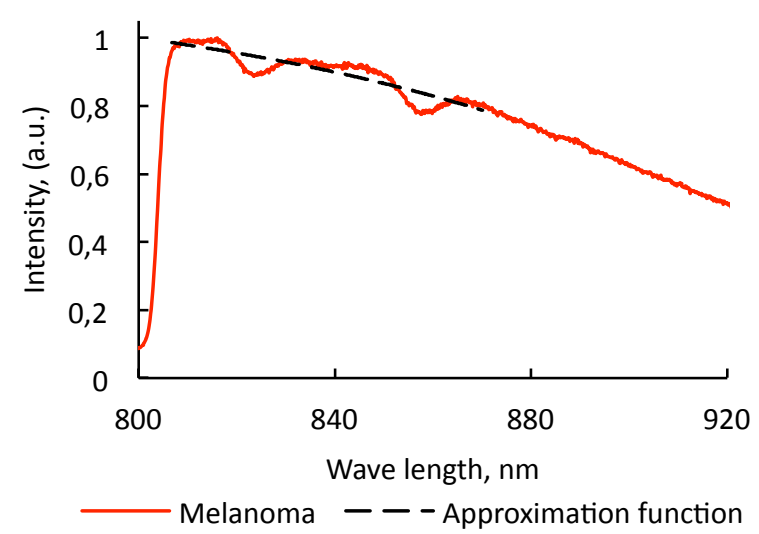

b) $a=-819.7 ; b=1.82$

Fig. 4 Exponential approximation of AF spectrum of basal cell carcinoma (a) and melanoma (b). 
Thus, as the informative criterion for the differentiation of tumour type we can consider the absolute value of the coefficient $|b|=\bar{b}$, that determines the curvature of the AF spectrum. It was found that the values of the coefficient $\bar{b}$ for melanoma are greater than 1 both for ex vivo and for in vivo measurements, whereas for other pathologies they are essentially smaller (see Fig. 5). Therefore, we can always introduce the criterial norm

$$
\bar{b} \geq 1
$$

that selects the melanoma among all possible neoplasms.

The second possible criterion for classifying the tumour type is the sign of the coefficient $a$ before the exponential function. From the analysis of the obtained experimental data, it follows that the coefficient $a$ is negative for all neoplasms with high concentration of melanin, in particular, for melanoma. Other types of tumours are characterised by positive values of $a$.

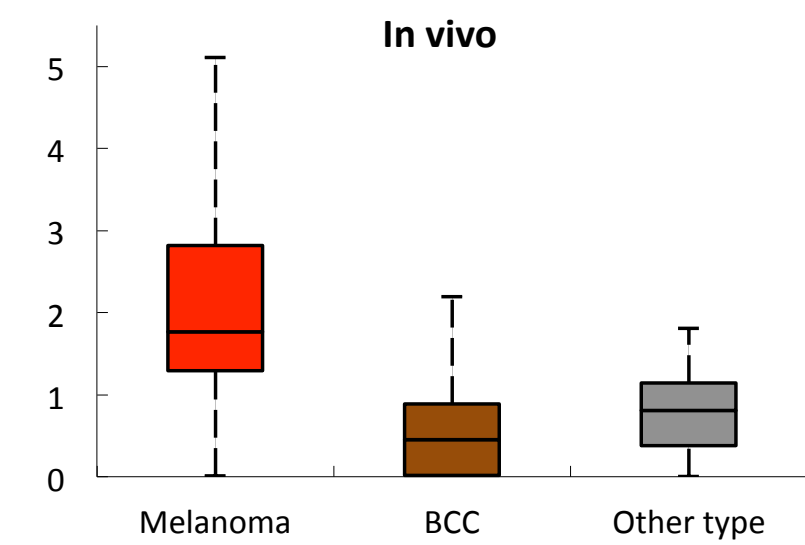

a)

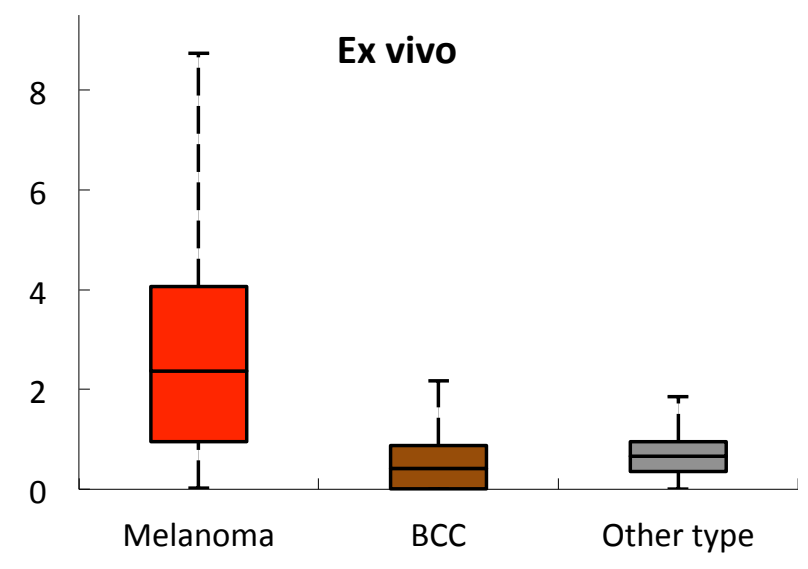

b)

Fig. 5 Classification of melanoma, basal cell carcinoma and other types of tumours based on the curvature $\bar{b}$ of the spectral curve for ex vivo (a) and in vivo (b) studies.
Table 1 presents the values of the sensitivity and specificity of the proposed method of diagnostics, calculated from the data obtained ex vivo and in vivo. Here the diagnostic precision of the method was defined as

$$
A C C=\frac{T P+T N}{T P+F P+F N+T N}
$$

where the terms in the right-hand side represent the number of true positive $T P$, false positive $F P$, true negative $T N$, and false negative $F N$ decisions.

In the case of using the criterion (5) for the melanoma selection among other types of the studied pathologies the values of the sensitivity and specificity reached $67 \%$ and $83 \%$ for ex vivo and $78 \%$ and $80 \%$ for in vivo studies, respectively. When a combination of both criteria ( $a$ and $\bar{b}$ ) was used, the result was considered positive when one of the criteria showed that the neoplasm is melanoma. In this case, the values of the sensitivity and specificity for the diagnostics of melanoma increase to $96 \%$ and $83 \%$ for ex vivo and $92 \%$ and $80 \%$ for in vivo studies. The relatively low value of the specificity is due to the fact that the proposed criteria of the spectral curve shape allow one to select the pathologies with high content of melanocytes, which is typical both for melanomas and for nevi. This lead to the identification of some nevi as melanomas.

Table 1 Accuracy of melanoma diagnostics.

\begin{tabular}{cccc}
\hline Ex vivo & $\begin{array}{c}\text { Sensitivity, } \\
\%\end{array}$ & $\begin{array}{c}\text { Specificity, } \\
\%\end{array}$ & $\begin{array}{c}\text { Diagnostic } \\
\text { accuracy, } \\
\%\end{array}$ \\
\hline$\overline{\boldsymbol{b}}$ & 67 & 83 & 75.5 \\
$\boldsymbol{a}$ & 64 & 66 & 65 \\
$\overline{\boldsymbol{b}}+a$ & 96 & 83 & 88.4 \\
\hline \multicolumn{4}{c}{} \\
\hline $\boldsymbol{I n}$ vivo & Sensitivity, & Specificity, & Diagnostic \\
& $\%$ & $\%$ & accuracy, \\
& & & $\%$ \\
\hline$\overline{\boldsymbol{b}}$ & 78 & 80 & 79 \\
$\boldsymbol{a}$ & 61 & 50 & 55.5 \\
$\overline{\boldsymbol{b}}+\boldsymbol{a}$ & 92 & 80 & 86.2 \\
\hline
\end{tabular}

The melanoma diagnostic accuracy values for $e x$ vivo $(75.5 \%)$ and in vivo $(79 \%)$ studies using the criterion $\bar{b}$ differ by $3.5 \%$. This difference can be related to the relatively small sensitivity of detecting the melanomas in the ex vivo measurements (67\%), because in the ex vivo measurements the number of melanoma samples was by 2.5 times greater than in the studies in vivo. On the other hand, when using the criterion $a$ the observed difference between the values of diagnostic accuracy for melanomas ex vivo (65\%) and in vivo $(55.5 \%)$ studies is more essential $(9.5 \%)$. This difference is explained by the low specificity $(50 \%)$ of the diagnostics for in vivo measurements that can be associated with the larger fraction of nevi and other 
non-melanoma pigmented neoplasms than in the ex vivo studies. Using the combination of both criteria of the AF spectrum shape allows the nearly similar accuracy of the melanoma diagnostics for ex vivo and in vivo cases at the level of $88.4 \%$ and $86.2 \%$, respectively. However, the combination of the proposed criteria of the AF spectra shape appeared to be low-informative in the identification of the basal cell carcinoma, benign neoplasms, and nevi.

Comparing the results of the performed study with the data of other authors on the melanoma diagnostics one can conclude that the proposed method based on the criteria of the spectral curve shape change is comparable in the accuracy of melanoma diagnostics to the multimodal method [21] with the excitation of autofluorescence in the near infrared spectral region. In Ref. [21] it is shown that the diagnostic accuracy in the case of using the AF method reaches $90 \%$ in differentiating melanoma from healthy skin, however, the efficiency sharply falls in differentiating melanoma from nevus, with the values of sensitivity $67 \%$ and specificity $18 \%$. Since both melanoma and nevus belong to the neoplasms of melanocytic type, their differentiation is difficult. In our work, we studied 3 nevi ex vivo and 3 nevi in vivo. Four of the six studied nevi were classified as melanoma basing on the proposed criteria, which is caused by the fact that both melanomas and nevi contain a large amount melanin fluorescing in the spectral region of interest. Apparently, this problem may be solved by operating in the visible or UV range [22, 23]. However, one cannot increase the accuracy of the melanoma diagnostics. Thus, according to the data of Ref. [23], the sensitivity and specificity of the melanoma diagnostics based on the measurement of the AF intensity under the UV laser excitation reached $77.8 \%$ and $93.3 \%$, respectively. Thus, the efficiency of the proposed method is comparable with that of the method of the melanoma $\mathrm{AF}$ analysis in the visible spectral range.

\section{Conclusions}

The results obtained in our experimental study show the possibility of using the AF spectrum shape analysis in the near infrared region for the malignant melanoma diagnostics.

An important advantage of this method is its invariance with respect to the tumour size and the high speed of the information acquisition.

The best results in the melanoma diagnostics are achieved using the complex shape criteria, applied to the autofluorescence spectrum approximated by the exponential function. The accuracy of selecting melanoma among other types of tumours achieves $88.4 \%$ for ex vivo studies and $86.2 \%$ for in vivo studies. However, the method appeared inefficient for the diagnostics of non-melanocytic types of malignant tumours. In that the malignant melanoma is the most aggressive and dangerous pathology, the obtained results can be important for the medical practice. Since the proposed method is characterised by high speed of processing the results and calculating the criterial norms and the method is independent of the fluorescence intensity level and the skin phenotype, it can be successfully applied in screening examinations. An alternative application is the fast primary instrumental testing with subsequent analysis of Raman scattering of the pathologies, detected at the first stage.

\section{Acknowledgments}

The work was supported by the Ministry of Education and Science of the Russian Federation. 